\title{
On the Convergence of Discrete Approximations to the Navier-Stokes Equations*
}

\author{
By Alexandre Joel Chorin
}

\begin{abstract}
A class of useful difference approximations to the full nonlinear Navier-Stokes equations is analyzed; the convergence of these approximations to the solutions of the corresponding differential equations is established and the rate of convergence is estimated.
\end{abstract}

Introduction. The Navier-Stokes equations, describing the motion of a viscous incompressible fluid, can be written in the dimensionless form

$$
\begin{aligned}
\partial_{t} v_{i}+\partial_{i} p & =-v_{j} \partial_{j} v_{i}+\nabla^{2} v_{i}+E_{i}, \quad\left(\nabla^{2} \equiv \sum \partial_{j}{ }^{2}\right) \\
\operatorname{div} \mathbf{v} & =0
\end{aligned}
$$

where the vector $\mathbf{v}$, with components $v_{i}, i=1,2,3$, is the velocity, $p$ is the pressure, $\mathbf{E}$ is the external force, $\partial_{t}$ denotes differentiation with respect to the time $t$ and $\partial_{i}$ denotes differentiation with respect to the space variable $x_{i}, i=1,2,3$. Vector quantities are denoted by bold-face characters and the summation convention applies to the index $j$.

When a solution of these equations is required in some bounded domain $\Omega$ with boundary $\partial \Omega$, use is generally made of an appropriate difference approximation. A new class of such approximations was introduced and utilized in [1] and [2]; it is the purpose of this paper to establish the convergence of the solutions of such approximations to the solutions of Eqs. (1) and (2) in $\Omega$.

To our knowledge, the first convergence proof for a difference approximation to the complete system (1) and (2) was given by Krzywicki and Ladyzhenskaya (see e.g. [3]). Their proof gives both more and less than the numerical analyst requires. It gives more because it actually establishes the existence of a certain weak solution of the equations. It gives less because it provides no estimate of the error and because it applies to a scheme which is not readily applied in practical calculation. Proofs related to that of Krzywicki and Ladyzhenskaya have been given by Temam [4], [5], for schemes which are as yet untested in practice.

In the present paper we shall adopt a different point of view. We shall assume that the differential equations have a solution with a certain number of continuous derivatives. Armed with this knowledge, we shall study difference schemes which are not merely usable, but even efficient. The methods analyzed are based on the following observations: Equation (1) can be written in the form

$$
\partial_{t} \mathrm{v}+\operatorname{grad} p=\mathfrak{F v}+\mathrm{E}
$$

Received September 12, 1968, revised November 3, 1968.

* This work was partially supported by the U.S. Atomic Energy Commission, Contract No. AT(30-1)-1480. 
where the vector $\mathfrak{F} \mathbf{v}$, with components $-v_{j} \partial_{j} v_{i}+\nabla^{2} v_{i}$, is a functional of $\mathbf{v}$; Eq. (2) can be differentiated to yield

$$
\operatorname{div}\left(\partial_{t} \mathbf{v}\right)=0 .
$$

$\left(1^{\prime}\right)$ can therefore be written in the form

$$
\partial_{t} \mathbf{v}=\rho(\mathfrak{F} \mathbf{v}+\mathbf{E})
$$

where $P$ is an orthogonal projection operator which projects vectors in $L_{2}(\Omega)$ onto the subspace of vectors with zero divergence in $\Omega$ and satisfying an appropriate boundary condition on $\partial \Omega$ (see e.g. [6], [7]). Usually the appropriate boundary condition is that the normal component of $\mathbf{v}$ vanishes. On the basis of these remarks the following procedure is followed: The time $t$ is discretized; at every time level $\mathfrak{F v}$, then $\odot(\mathfrak{F} \mathbf{v}+\mathbf{E})$ are evaluated; this yields an approximation to $\partial_{t} \mathbf{v}$ which is used to obtain $\mathbf{v}$ at the next time level.

As will become apparent in the course of this work, the author has not obtained results as general as he may have wished. A convergence proof in both the maximum and $L_{2}$ norms, with a suitable error estimate, has been obtained only for the special problems in which the boundary conditions are replaced by periodicity conditions. This proof is presented in the next two sections; first the discrete analogues of the operators grad, div and $\odot$ are described and studied; these operators are then used to present and analyze a difference scheme for the periodic initial value problem. The mixed initial value-boundary value problem is briefly discussed in a final section.

Preliminaries; The Operators $D, \mathrm{G}$ and $P$. We assume that Eqs. (1) and (2) have a solution $\nabla, p$, periodic in all spatial directions; without loss of generality in the proofs the periods can be taken equal to 1 . Let $l$ be the number of space dimensions; $\Omega$ is then the cube $0 \leqq x_{i} \leqq 1, i=1, \cdots, l$. We cover $\Omega$ by a rectangular grid and assume that the mesh-widths in all directions are equal to the same small number $h$. The set of all mesh-nodes is denoted by $\Omega_{h} ; \Omega_{h}{ }^{0}$ is the set of nodes in the interior of $\Omega$ and $\partial \Omega_{h}$ is the set of nodes on the boundary of $\Omega_{.} \Omega_{h}^{0}+\partial \Omega_{h}=\Omega_{h}$. $N=h^{-1}+1$ is the number of mesh-points in each space direction.

Let $f$ be a scalar function and let $\mathfrak{u}$ be a vector function with components $u_{i}$, defined at the points of $\Omega_{h}$. Let $z=[q, r]$ (if $l=2$ ) or $z=[q, r, s]$ (if $l=3$ ) be a point in $\Omega_{h}$ with coordinates $q h, r h(q h, r h$, sh if $l=3)$. The values of $f, u_{i}$ at $z$ are denoted by $f_{z}, u_{i(z)}$ or $f_{q, r}, u_{i(q, r)}\left(f_{q, r, s}, u_{i(q, r, s)}\right.$, if $\left.l=3\right)$. The periodicity conditions become $f_{q+N-1, r}=f_{q, r}$ etc.

The inner product is defined for scalar functions $f, g$, by

$$
(f, g)=\sum_{z \in \Omega_{h}^{0}} f_{z} g_{z} h^{l}+\frac{1}{2} \sum_{z \in \partial \Omega_{h}} f_{z} g_{z} h^{l}
$$

and for vectors $\mathbf{u}, \mathbf{v}$ by

$$
(\mathbf{u} \cdot \mathbf{v})=\sum_{i=1}^{l}\left(u_{i}, v_{i}\right)
$$

where only half the vertices are counted in the boundary sums. As usual, we set

$$
\|f\|=((f, f))^{1 / 2}, \quad\|\mathbf{u}\|=((\mathbf{u}, \mathfrak{u}))^{1 / 2} .
$$


The shift operators $S_{ \pm i}$ are defined by

$$
\begin{aligned}
& S_{ \pm 1} f_{q, r}=f_{q \pm 1, r} \\
& S_{ \pm 2} f_{q, r}=f_{q, r_{ \pm 1}}
\end{aligned}
$$

with similar definitions in the three-dimensional case. The difference operators $D_{+i}, D_{-i}, D_{0 i}$ are defined by

$$
\begin{aligned}
D_{+i} & =\left(S_{+i}-I\right) / h, \\
D_{-i} & =\left(I-S_{-i}\right) / h, \\
D_{0 i} & =\left(D_{+i}+D_{-i}\right) / 2=\left(S_{+i}-S_{-i}\right) / 2 h,
\end{aligned}
$$

where $I$ is the identity. $D_{+i}, D_{-i}$ and $D_{0 i}$ are respectively the forward, backward and centered difference operators in the $i$ th direction.

Let $D$ and $\mathbf{G}$ denote respectively the discrete approximations to the operators div and grad. Both $D$ and $\mathbf{G}$ employ centered differences, i.e. for a vector $\mathfrak{u}$ on $\Omega_{h}$ we set $D \mathfrak{u} \equiv D_{0 j} u_{j}$ and for a scalar function $\phi$ we set $G_{i} \phi \equiv D_{0 i} \phi$. With these definitions, the following identities can be readily verified:

$$
(D \mathbf{u}, e)=0,
$$

where $e \equiv 1$ at all points of $\Omega_{h}$, and

$$
(D \mathbf{u}, \phi)+(\mathbf{u}, \mathbf{G} \phi)=0
$$

for all $\mathbf{u}$ and $\phi$. These are the analogues of the identities

$$
\int_{\Omega} \operatorname{div} u d x=0
$$

and

$$
\int_{\Omega} \phi \operatorname{div} \mathbf{u} d x+\int_{\Omega} \mathbf{u} \operatorname{grad} \phi d x=\int_{\Omega} \operatorname{div} \phi \mathbf{u} d x=0
$$

which hold for smooth periodic functions $\mathbf{u}$ and $\phi$ on $\Omega$. For $\mathbf{u}, \phi$ periodic and three times continuously differentiable, we have

$$
\|\mathbf{G} \phi-\operatorname{grad} \phi\|=O\left(h^{2}\right), \quad\|D \mathbf{u}-\operatorname{div} \mathbf{u}\|=O\left(h^{2}\right) .
$$

We shall now discuss some consequences of our systematic use of centered differences. Let $\psi$ be a function on $\Omega_{h}$, let $z_{-}, z_{+}$be two points a distance $2 h$ (modulo 1 ) apart, and let $z_{0}$ be the point on the line joining $z_{-}$and $z_{+}$and at a distance $h$ from each. One of the components of $\mathbf{G} \psi$ at $z_{0}$ is a linear combination of $\psi_{z_{+}}$and $\psi_{z_{-}}$; we describe this situation by saying the $z_{+}$and $z_{-}$are $G$-connected. We say that points $z, z^{\prime}$ belong to the same $G$-chain if there exist points $z_{1}, z_{2}, \cdots, z_{n}$ such that any two successive points in the sequence

$$
z, z_{1}, z_{2}, \cdots, z_{n}, z^{\prime}
$$

are $G$-connected. Clearly $\Omega_{h}$ is the union of some number $L$ of disjoint $G$-chains. If $N$ is even $L=1$; if $N$ is odd $L=2^{l}$. (Had we allowed the numbers of mesh-points in the several space directions to differ from each other, we would have found that 
$L=2^{i}, i$ between 0 and $l$.) The following facts can now be verified: (i) when $\mathbf{G}_{\phi}$ is given, $\phi$ is determined only up to $L$ arbitrary constants; and (ii) the sum on the left-hand side of the identity (4) can be separated into $L$ partial sums, each vanishing separately. We refrain from assuming that $N$ is even and $L=1$ so that our discussion remain valid for the nonperiodic case where $L \neq 1$ for all $N$; see the last section of this paper.

We are now ready to prove the following discrete analogue of a well-known decomposition theorem:

Theorem 1. Let $\mathbf{u}$ be a vector on $\Omega_{h}$ satisfying the periodicity conditions; then there exist a unique periodic vector $\mathbf{u}^{D}$ and a periodic function $\phi$ such that

$$
\begin{aligned}
& D \mathbf{u}^{D}=0 \\
& \mathbf{u}=\mathfrak{u}^{D}+\mathbf{G}_{\boldsymbol{\phi}}
\end{aligned}
$$

at all points of $\Omega_{h}$, with

$$
\left(\mathbf{u}^{D}, \mathbf{G} \phi\right)=0 .
$$

Proof. If $\mathbf{u}^{D}$ satisfying Eq. (6) exists, then Eq. (8) is clearly satisfied because $\left(\mathbf{u}^{D}, \mathbf{G} \phi\right)=-\left(D \mathbf{u}^{D}, \phi\right)=0$.

We already know that $\phi$ in Eq. (7) can be determined only up to $L$ arbitrary constants. To lift this indeterminacy we can impose $L$ additional conditions; for example, we can number the $G$-chains and require that

$$
\sum_{i} \phi_{z}=0, \quad i=1, \cdots, L,
$$

where $\sum_{i}$ denotes summation over the $i$ th $G$-chain.

The theorem is proved by verification of the Fredholm alternative. Let $q_{0}$ be the number of points in $\Omega_{h}{ }^{0}$ and $q_{\partial}$ the number of points on $\partial \Omega_{h}\left(q_{0}+q_{\partial}=N^{l}\right)$. There are $q_{0}+q_{\partial} / 2$ values of $\phi$ and $l\left(q_{0}+q_{\partial} / 2\right)$ components of $\mathbf{u}^{D}$ to determine. Equation (6) represents $\left(q_{0}+q_{\partial} / 2\right)$ equations related by the $L$ identities (4), i.e. $q_{0}+q_{\partial} / 2-L$ independent equations. Equation (7) represents $l\left(q_{0}+q_{\partial} / 2\right)$ relations; together with Eqs. (9) the number of equations equals the number of unknowns.

Squaring (7) and using (8) we obtain

$$
\|\mathbf{u}\|^{2}=\left\|\mathbf{u}^{D}\right\|^{2}+\|\mathbf{G} \phi\|^{2} .
$$

Therefore, if $\mathbf{u}=0$, then $\mathbf{u}^{D}=0, \mathbf{G} \phi=0$ and $\phi=0$; this proves the theorem.

Let $H$ be the space of periodic vectors defined on $\Omega_{h}$, let $H_{D}$ be the subspace of periodic vectors $\mathbf{v}$ such that $D \mathbf{v}=0$, and let $H_{G}$ be the subspace of vectors of the form $\mathbf{G} \phi, \phi$ periodic; Theorem 1 states that $H_{G}$ and $H_{D}$ are orthogonal to each other, and that $H$ is their direct sum. Let $P$ be the orthogonal projection projecting $H$ on $H_{D}$; (7) can be written in the form

$$
\mathfrak{u}=P \mathfrak{u}+\mathbf{G} \phi .
$$

We obviously have for all $\mathbf{u}$

$$
\|P \mathbf{u}\| \leqq\|\mathbf{u}\| .
$$

$P$ is the discrete analogue of $\rho$ (see Eq. (3)). Given $\mathbf{u}$, it is a fairly simple matter to 
evaluate $P \mathfrak{u}$; efficient methods for so doing were described in [2]. For the sake of completeness, we exhibit a method for finding $P \mathbf{u}$ which, albeit inefficient, has the merit of simplicity. Consider the iteration system

$$
\begin{aligned}
\mathbf{w}^{m+1} & =\mathbf{u}-\mathbf{G} \phi^{m} \quad m \geqq 1, \\
\phi^{m+1} & =\phi^{m}-\theta D \mathbf{w}^{m+1} \quad m \geqq 1, \quad \theta>0
\end{aligned}
$$

where $\mathbf{w}^{m}, \phi^{m}, m \geqq 1$, are periodic and $\theta$ is a parameter. It is readily verified that for $0<\theta<h^{2} / l^{2}$, w $\mathbf{w}^{m}$ converges to $P \mathfrak{u}$ as $m$ increases for all initial guesses $\phi^{1}$.

To conclude this section, we prove a number of inequalities which will be needed in later sections. We start with a discrete analogue of the Poincaré inequality. Consider the case $l=2$. Let $\psi$ be a function defined on $\Omega_{h}$, and let $z=[p, q], z^{\prime}=\left[p^{\prime}, q^{\prime}\right]$ be two points on the $i$ th $G$-chain; $p^{\prime}=p+2 m_{1}, q^{\prime}=q+2 m_{2}$. We have,

$$
\psi_{p^{\prime}, q^{\prime}}-\psi_{p, q}=\sum_{k=0}^{m-1}\left(G_{1} \psi_{p+1+2 k, q} \cdot 2 h+\sum_{k=0}^{m_{2}-1}\left(G_{2} \psi\right)_{p^{\prime}, q+1+2 k} \cdot 2 h .\right.
$$

Therefore

$$
\begin{aligned}
\left|\psi_{p^{\prime}, q^{\prime}}-\psi_{p, q}\right|^{2} & \leqq 4\left[\sum_{k=1}^{N-1}\left|G_{1} \psi\right|_{k, q} h+\sum_{k=1}^{N-1}\left|G_{2} \psi\right|_{p^{\prime}, k} h\right]^{2} \\
& \leqq 8\left[\sum_{k=1}^{N-1}\left|G_{1} \psi\right|_{k, q}^{2} h+\sum_{k=1}^{N-1}\left|G_{2} \psi\right|_{p^{\prime}, k}^{2} h\right],
\end{aligned}
$$

where the relation $(N-1) h=1$ is used. We multiply both sides by $h^{4}$ and sum over all $[p, q]$ and $\left[p^{\prime}, q^{\prime}\right]$ in the $i$ th $G$-chain, giving points on $\partial \Omega_{h}$ the weight $\frac{1}{2}$, and obtain

$$
\begin{gathered}
\sum_{i} h^{2} \sum_{i}|\psi|_{p, q}^{2} h^{2}+\sum_{i} h^{2} \sum_{i}|\psi|_{p^{\prime}, q^{\prime}}^{2} h^{2}-2 \sum_{i} \psi_{p, q} h^{2} \sum_{i} \psi_{p^{\prime}, q^{\prime}} h^{2} \\
\leqq 8 \sum_{i} h^{3}\|\mathbf{G} \psi\|^{2} \leqq 8\|\mathbf{G} \psi\|^{2}
\end{gathered}
$$

where $\sum_{i}$ denotes summation over the $i$ th $G$-chain.

Let $N_{i}$ be the number of points in the $i$ th $G$-chain. We have

$$
N_{i} \geqq(N-1)^{2} / L>\frac{1}{2} N^{2} / L \text {. }
$$

Therefore

$$
\sum_{i} h^{2} \geqq \frac{1}{2 L}
$$

and

$$
\frac{1}{L} \sum_{i}|\psi|_{p, q} h^{2} \leqq 2\left(\sum_{i} \psi_{p, q} h^{2}\right)^{2}+8\|\mathrm{G} \psi\|^{2} .
$$

Summing over all $G$-chains we obtain

$$
\|\psi\|^{2} \leqq 2 L \sum_{G \text {-chains }}\left(\sum_{i} \psi_{p, q} h^{2}\right)^{2}+C_{1}\|\mathbf{G} \psi\|^{2}
$$

where $C_{1}=8 L^{2}$. A similar inequality can be derived in the three-dimensional case, with $C_{1}=12 L^{2}$. The inequality (12) can now be used to prove the following theorem: 
Theorem 2. Let $\mathbf{u}$ be a periodic vector defined on $\Omega_{h}$. Then the following inequality holds

$$
\|\mathbf{u}-P \mathbf{u}\| \leqq \sqrt{ } C_{1}\|D \mathbf{u}\|,
$$

where $\sqrt{ } C_{1}$ is a constant independent of $\mathbf{u}$ and $h$.

Proof. By Theorem $1, \mathbf{u}-P \mathbf{u}=\mathbf{G} \phi$ is in $H_{G}$. Let $\mathbf{G} \psi$ be an arbitrary unit vector in $H_{G},(\|\mathbf{G} \psi\|=1) . \psi$ is determined only up to $L$ arbitrary constants which can be chosen so that

$$
\sum_{i} \psi_{z}=0, \quad i=1, \cdots, L
$$

We have

$$
(\mathbf{G} \phi, \mathbf{G} \psi)=(\mathbf{u}-P \mathfrak{u}, \mathbf{G} \psi)=(\mathbf{u}, \mathbf{G} \psi)=-(D \mathbf{u}, \psi) .
$$

Hence, using (12), we obtain

$$
|(\mathbf{G} \phi, \mathbf{G} \psi)|=|(D \mathbf{u}, \psi)| \leqq\|D \mathbf{u}\|\|\psi\| \leqq \sqrt{ } C_{1}\|D \mathbf{u}\| .
$$

Since $\mathbf{G} \psi$ is an arbitrary unit vector in $H_{G}$, (13) follows.

Solution of the Periodic Initial-Value Problem. In this section a scheme for finding periodic solutions of Eqs. (1) and (2) will be analyzed. The particular scheme discussed has been singled out because it resembles schemes the author has used in actual computation (see [2]); it will be evident that the analysis applies to wide classes of schemes. We shall again simplify notations by writing the equations for the two-dimensional case; the scheme as well as the proofs generalize to the three-dimensional case without further ado.

Let $\mathbf{u}$, with components $u_{i}$, be the computed velocity, let $\pi$ be the computed pressure, and let $k$ be the time step. We write

$$
\mathbf{u}^{n} \equiv \mathbf{u}(n k), \quad \pi^{n} \equiv \pi(n k), \quad \text { etc. }
$$

At the time $t=0$ a periodic velocity field $\mathbf{u}^{0}$ is assumed given. (More will be said later about the proper choice of $\mathbf{u}^{0}$.) Given $\mathbf{u}^{n}, \mathbf{u}^{n+1}$ is evaluated in three fractional steps:

$$
\begin{aligned}
u_{i}^{n+1 / 3} & =u_{i}^{n}-k u_{1}^{n} D_{01} u_{i}^{n+1 / 3}+k D_{+1} D_{-1} u_{i}{ }^{n+1 / 3} \\
u_{i}{ }^{n+2 / 3} & =u_{i}{ }^{n+1 / 3}-k u_{2}{ }^{n} D_{02} u_{i}{ }^{n+2 / 3}+k D_{+2} D_{-2} u_{i}{ }^{n+2 / 3} \\
\mathfrak{u}^{n+1} & =P\left(\mathbf{u}^{n+2 / 3}+k \mathbf{E}^{n+1}\right)
\end{aligned}
$$

with $\mathbf{u}^{n+1 / 3}, \mathbf{u}^{n+2 / 3}$ periodic.

Equations (14a) and (14b) can be rewritten in the form

$$
\begin{aligned}
& \left(I-k Q_{1}\left(\mathbf{u}^{n}\right)\right) \mathbf{u}^{n+1 / 3}=\mathbf{u}^{n}, \\
& \left(I-k Q_{2}\left(\mathbf{u}^{n}\right)\right) \mathbf{u}^{n+2 / 3}=\mathbf{u}^{n+1 / 3},
\end{aligned}
$$

where $Q_{1}\left(\mathbf{u}^{n}\right), Q_{2}\left(\mathfrak{u}^{n}\right)$, are linear operators dependent on the parameters $u_{i(z)}^{n}$. Equation (14c) can be rewritten in the form

$$
\mathbf{u}^{n+1}+k \mathbf{G} \pi^{n+1}=\mathbf{u}^{n+2 / 3}+k \mathbf{E}^{n+1} \quad\left(D \mathbf{u}^{n+1}=0\right)
$$


which defines $\pi^{n+1}$, the computed pressure at the time $(n+1) k$. ( $\mathbf{u}^{n+2 / 3}$ corresponds to $\mathbf{u}^{\text {aux }}$ in the notations of [1] and [2].) It can be seen that the vector $\left(\mathbf{u}^{n+2 / 3}-\mathbf{u}^{n}\right) / k$ approximates $\mathfrak{F} \mathbf{u}$ and that Eq. (14c) which is equivalent to

$$
\left(\mathbf{u}^{n+1}-\mathbf{u}^{n}\right) / k=P\left\{\left(\mathbf{u}^{n+2 / 3}-\mathbf{u}^{n}\right) / k+\mathbf{E}\right\}
$$

is the discrete analogue of Eq. (3).

The task now at hand is to prove that $\mathbf{u}^{n+1 / 3}, \mathfrak{u}^{n+2 / 3}, \mathbf{u}^{n+1}$ exist, i.e. that the operators $\left(I-k Q_{i}\left(\mathbf{u}^{n}\right)\right)$ are invertible when $\mathbf{u}^{0}$ is chosen appropriately, and that the vectors $\mathbf{u}^{n}$ converge to the solution $\mathbf{v}(n k)$ of Eqs. (1) and (2). We start by showing that Eqs. (14) are consistent with Eqs. (1) and (2); this is the content of the following lemma:

Lemma 1. Let $k=O\left(h^{2}\right)$, and assume that Eqs. (1) and (2) have a periodic solution $\mathbf{v}, p$, which has continuous derivatives up to order five in the interval $0 \leqq t \leqq T$. Then there exist two times continuously differentiable vectors $\mathbf{w}^{n}, \mathbf{w}^{n+1 / 3}, \mathbf{w}^{n+2 / 3}(0 \leqq n k<T)$ such that

$$
\begin{aligned}
\left(I-k Q_{1}\left(\mathbf{w}^{n}\right)\right) \mathbf{w}^{n+1 / 3} & =\mathbf{w}^{n}+O\left(k^{2}\right), \\
\left(I-k Q_{2}\left(\mathbf{w}^{n}\right)\right) \mathbf{w}^{n+2 / 3} & =\mathbf{w}^{n+1 / 3}+O\left(k^{2}\right), \\
\mathbf{w}^{n+1} & =P\left(\mathbf{w}^{n+2 / 3}+k \mathbf{E}^{n+1}\right)+O\left(k^{2}\right),
\end{aligned}
$$

with

$$
\left\|\mathbf{v}^{n}-\mathbf{w}^{n}\right\|=O(k) .
$$

Proof. We simply construct the required functions. We have

$$
D \mathbf{v}^{n}=\operatorname{div} \mathbf{v}^{n}+\sum_{\beta=1}^{l} \frac{h^{2}}{3 !} \partial_{\beta}^{3} v_{\beta}^{n}+O\left(h^{4}\right) .
$$

Therefore, putting

$$
w_{\beta}{ }^{n}=v_{\beta}{ }^{n}-\frac{h^{2}}{3 !} \partial_{\beta}{ }^{2} v_{\beta}{ }^{n} \quad \beta=1, \cdots, l(\text { no summation over } \beta)
$$

we obtain $D \mathbf{w}^{n}=O\left(h^{4}\right)=O\left(k^{2}\right)$. Equation (17) is clearly satisfied, and by Theorem 2

$$
\left\|\mathbf{w}^{n}-P \mathbf{w}^{n}\right\|=O\left(k^{2}\right) \text {. }
$$

We now put

$$
\begin{aligned}
& w_{i}^{n+1 / 3}=w_{i}^{n+1}-k \partial_{2}^{2} v_{i}^{n+1}+k v_{2}^{n} \partial_{2} v_{i}^{n+1}+k \partial_{i} p^{n+1}-k E_{i}^{n+1} \\
& w_{i}^{n+2 / 3}=w_{i}^{n+1}+k \partial_{i} p^{n+1}-k E_{i}^{n+1} .
\end{aligned}
$$

Equations (16a) and (16b) are clearly satisfied, and since

$$
\mathbf{G} p=\operatorname{grad} p+O\left(h^{2}\right)=\operatorname{grad} p+O(k)
$$

we have

$$
P(k \operatorname{grad} p)=O\left(k^{2}\right) .
$$

On the other hand, it can always be assumed that $\operatorname{div} \mathrm{E}=0$. (Since adding a gradient to $\mathbf{E}$ merely changes the definition of $p$; see e.g. [7]), and therefore by Theorem 2 


$$
\|k \mathrm{E}-k P \mathrm{E}\| \leqq \sqrt{ } C_{1} k\|D \mathrm{E}\|=O\left(k^{2}\right) .
$$

These equations, together with Eq. (19) show that (16c) is satisfied, and the lemma is proved.

We shall use $\mathbf{w}^{n}$ as a comparison vector, i.e. we shall prove that $\left\|\mathbf{u}^{n}-\mathbf{w}^{n}\right\|$ is small, and use (17) at the end of the argument to show that $\left\|\mathbf{u}^{n}-\nabla^{n}\right\|$ is small. The lemma assumes that $\mathbf{v}$ has continuous derivatives up to order five. Had we assumed the existence of only four continuous derivatives, the error term in (16c) would have been of order $k h$. This is sufficient for convergence; however, the proof becomes somewhat more complicated and we shall be content with the assumption of the lemma.

We now introduce a second norm, the discrete maximum norm, defined for scalar functions $\phi$ by

$$
\|\phi\|_{\max }=\max _{z \in \Omega_{h}}\left|\phi_{z}\right|
$$

and for vectors $\mathbf{u}$ by

$$
\|\mathfrak{u}\|_{\max }=\max _{i}\left\|u_{i}\right\|_{\max }
$$

We have

LEMma 2. $\|\phi\|_{\max } \leqq h^{-l / 2}\|\phi\|,\|\mathbf{u}\|_{\max } \leqq h^{-l / 2}\|\mathbf{u}\|$.

The proof is obvious from the definitions. This lemma is crucial to the sequel since, as we shall see, it implies that if $\mathbf{u}$ converges to $\mathbf{v}$ with sufficient accuracy in the $L_{2}$ norm, then $\mathbf{u}$ also converges to $\mathbf{v}$ in the maximum norm.

Lemma 3. Let $\left\|\mathbf{u}^{n}\right\|_{\max } \leqq K$, and let $k$ be small enough for the inequality $k K^{2} / 4<1$ to hold. Equations (14a) and (14b) can then be uniquely solved for $\mathfrak{u}^{n+1 / 3}, \mathbf{u}^{n+2 / 3}$.

Proof. Multiplying (14a) by $u_{i}^{n+1 / 3}$ we obtain

$$
\begin{aligned}
\left\|u_{i}^{n+1 / 3}\right\|^{2}= & -k\left(u_{i}^{n+1 / 3}, u_{1}^{n} D_{01} u_{i}^{n+1 / 3}\right) \\
& +k\left(u_{i}^{n+1 / 3}, D_{+1} D_{-1} u_{i}^{n+1 / 3}\right) \\
& +\left(u_{i}^{n}, u_{i}^{n+1 / 3}\right),
\end{aligned}
$$

however, we have

$$
\begin{aligned}
\left(u_{i}^{n+1 / 3}, D_{+1} D_{-1} u^{n+1 / 3}\right) & =-\left\|D_{+1} u_{i}^{n+1 / 3}\right\|^{2} \\
\left|\left(u_{i}{ }^{n+1 / 3}, u_{1}^{n} D_{01} u_{i}^{n+1 / 3}\right)\right| & \leqq\left\|u_{1}^{n}\right\|_{\max }\left\|u_{i}{ }^{n+1 / 3}\right\|\left\|D_{01} u_{i}{ }^{n+1 / 3}\right\| .
\end{aligned}
$$

On the other hand, $D_{01}=\frac{1}{2}\left(D_{+1}+D_{-1}\right)$, therefore

$$
\left\|D_{01} u_{i}^{n+1 / 3}\right\| \leqq \frac{1}{2}\left(\left\|D_{+1} u_{i}^{n+1 / 3}\right\|+\left\|D_{-1} u_{i}^{n+1 / 3}\right\|\right)=\left\|D_{+1} u_{i}^{n+1 / 3}\right\|
$$

and

$$
\begin{aligned}
\left|k\left(u_{i}^{n+1 / 3}, u_{1}^{n} D_{01} u_{i}^{n+1 / 3}\right)\right| & \leqq K k\left\|u_{i}^{n+1 / 3}\right\|\left\|D_{+1} u_{i}^{n+1 / 3}\right\| \\
& \leqq k\left\|D_{+1} u_{i}^{n+1 / 3}\right\|^{2}+\left(K^{2} k / 4\right)\left\|u_{i}^{n+1 / 3}\right\|^{2},
\end{aligned}
$$

and hence

$$
\left\|u_{i}^{n+1 / 3}\right\|\left(1-\frac{k K^{2}}{4}\right) \leqq\left\|u_{i}{ }^{n}\right\|
$$


The existence and uniqueness of $u_{i}{ }^{n+1 / 3}$ follow by the Fredholm alternative. The existence and uniqueness of $u_{i}{ }^{n+2 / 3}$ are established in the same way.

Lemma 4. Let $\left\|\mathbf{u}^{n}\right\|_{\max } \leqq K$, and let $k$ be small enough for the inequality $k K^{2} / 2<1$ to hold; then, if $k=O\left(h^{2}\right)$, we have

$$
\left\|\mathbf{u}^{n+1}-\mathbf{w}^{n+1}\right\| \leqq\left(1+k C_{2}(K)\right)\left\|\mathbf{u}^{n}-\mathbf{w}^{n}\right\|+C_{3} k h^{2},
$$

where $C_{3}$ is a constant, and $C_{2}(K)$ is a constant whose magnitude depends on $K$.

Proof. Subtracting (14a) from (16a) we obtain

$$
\begin{aligned}
u_{i}^{n+1 / 3}-w_{i}{ }^{n+1 / 3}= & -k u_{1}^{n} D_{01}\left(u_{i}{ }^{n+1 / 3}-w_{i}{ }^{n+1 / 3}\right)-k\left(u_{1}{ }^{n}-w_{1}{ }^{n}\right) D_{01} w_{i}{ }^{n+1 / 3} \\
& +k D_{+1} D_{-1}\left(u_{i}^{n+1 / 3}-w_{i}{ }^{n+1 / 3}\right)+O\left(k^{2}\right) .
\end{aligned}
$$

Multiplication by $u_{i}{ }^{n+1 / 3}-w_{i}{ }^{n+1 / 3}$ and manipulations similar to those in the proof of Lemma 3 yield

$$
\left\|u_{i}^{n+1 / 3}-w_{i}^{n+1 / 3}\right\|\left(1-\frac{k K^{2}}{4}\right) \leqq\left\|u_{i}^{n}-w_{i}^{n}\right\|\left(1+k M_{1}\right)+O\left(k^{2}\right),
$$

where

$$
M_{1}=\max _{i} \max _{0 \leqq t \leqq T} \max _{\Omega}\left|\partial_{1} w_{i}^{n+1 / 3}\right|
$$

Similarly, we obtain

$$
\left\|u_{i}^{n+2 / 3}-w_{i}^{n+2 / 3}\right\|\left(1-\frac{k K^{2}}{4}\right) \leqq\left\|u_{i}^{n+1 / 3}-w_{i}^{n+1 / 3}\right\|+k M_{2}\left\|u_{i}^{n}-w_{i}{ }^{n}\right\|+O\left(k^{2}\right),
$$

where

$$
M_{2}=\max _{i} \max _{0 \leqq t \leqslant T} \max _{\Omega}\left|\partial_{2} w_{i}^{n+2 / 3}\right|
$$

and hence

$$
\left\|\mathbf{u}^{n+2 / 3}-\mathbf{w}^{n+2 / 3}\right\| \leqq\left(1+k C_{2}(K)\right)\left\|\mathbf{u}^{n}-\mathbf{w}^{n}\right\|+O\left(k^{2}\right),
$$

where $C_{2}(K)$ depends on $K$. Finally

$$
\mathbf{u}^{n+1}-\mathbf{w}^{n+1}=P\left(\mathbf{u}^{n+2 / 3}-\mathbf{w}^{n+2 / 3}\right)+O\left(k^{2}\right)
$$

and, therefore, using (11)

$$
\begin{aligned}
\left\|\mathbf{u}^{n+1}-\mathbf{w}^{n+1}\right\| & \leqq\left\|\mathbf{u}^{n+2 / 3}-\mathbf{w}^{n+2 / 3}\right\|+O\left(k^{2}\right) \\
& \leqq\left(1+k C_{2}(K)\right)\left\|\mathbf{u}^{n}-\mathbf{w}^{n}\right\|+C_{3} k h^{2}
\end{aligned}
$$

and the lemma is proved.

Let $\mathbf{u}^{0}$ be the initial value of $\mathbf{u}$, for use in Eqs. (14). We assume that

$$
\left\|\mathbf{u}^{0}-\mathbf{w}^{0}\right\|=C_{4} h^{2} .
$$

This can be achieved for example by putting $\mathbf{u}^{0}=\mathbf{v}^{0}$. Let $W$ be defined by

$$
W=\max _{i} \max _{0 \leqq t \leqq T} \max _{\Omega} \mid w_{i \mid}
$$


Let $C$ be the largest of $C_{3}, C_{4}$ and $C_{2}(2 W)$. Assume $k$ is so small that $k C^{\prime}<<1$ and that $h$ is smaller than $h_{0}$, where

$$
\max (C, 1) h_{0}{ }^{(4-l) / 2}=\frac{1}{2} \epsilon W, \quad \epsilon<1 .
$$

Equation (22) and Lemma 2 then show that

$$
\left\|\mathbf{u}^{0}\right\|_{\max } \leqq W+\frac{1}{2} \epsilon W<2 W .
$$

By Lemma $3 \mathbf{u}^{1}$ exists and by Lemma 4 we have $\left\|\mathbf{u}^{1}-\mathbf{w}^{1}\right\| \leqq(1+C k) C h^{2}+C k h^{2}$. Therefore

$$
\left\|\mathbf{u}^{1}-\mathbf{w}^{1}\right\|_{\max } \leqq \frac{\epsilon}{2}(1+C k) W+\frac{\epsilon}{2} k W<\epsilon W<W, \quad\left\|\mathbf{u}^{1}\right\|_{\max }<2 W,
$$

and we can evaluate $\mathbf{u}^{2}$. In general we have

$$
\begin{aligned}
\left\|\mathbf{u}^{n+1}-\mathbf{w}^{n+1}\right\| & \leqq(1+C k)^{n+1} C h^{2}+\left[1+(1+C k)+\cdots+(1+C k)^{n}\right] C k h^{2} \\
& <2 e^{C(n+1) k} \max (C, 1) h^{2}
\end{aligned}
$$

and

$$
\left\|\mathbf{u}^{n+1}-\mathbf{w}^{n+1}\right\|_{\max } \leqq \epsilon W e^{C t}, \quad(t=(n+1) k) .
$$

Let $T_{0}$ be defined by $\exp C T_{0}=1 / \epsilon$ and let $T_{1}=\min \left(T, T_{0}\right)$. Inequality (24) shows that for $0 \leqq t \leqq T_{1},\|\mathbf{u}\|_{\max } \leqq 2 W$ and hence for $0 \leqq n k<T_{1}, \mathbf{u}^{n+1}$ exists and

$$
\left\|\mathbf{u}^{n+1}-\mathbf{w}^{n+1}\right\| \leqq 2 \max (C, 1) e^{C t} h^{2}
$$

as well as

$$
\left\|\mathbf{u}^{n+1}-\mathbf{w}^{n+1}\right\|_{\max } \leqq 2 \max (C, 1) e^{C t} h^{(4-l) / 2} .
$$

If $T_{1}<T$, i.e. if the inequalities (25) hold for a time interval shorter than the time interval for which the solution of the differential equations has five bounded derivatives and for which a numerical solution is required, the above process can be restarted at $t=T_{1}$, to yield convergence for the whole finite interval $0 \leqq t \leqq T$.

Bearing in mind the definition of $\mathbf{w}$ and Eq. (17), we obtain the following theorem:

Theorem 3. Let Eqs. (1) and (2) have a periodic solution with continuous derivatives up to order five for $0 \leqq t \leqq T$. Let $k=O\left(h^{2}\right) ;$ if $\left\|\mathbf{u}^{0}-\mathbf{w}^{0}\right\|, k$ and $h$ are sufficiently small, Eqs. (14) have a unique solution which converges to the solution of (1) and (2) in both the $L_{2}$ and maximum norms. The error in the $L_{2}$ norm is of order $h^{2}$; the error in the maximum norm is bounded by $O(h)$ in the two-dimensional case and by $O(\sqrt{ } h)$ in the three-dimensional case.

Theorem 3 and its proof can be summarized as follows: Let $\mathbf{u}_{z}{ }^{n}, \mathbf{w}_{z}{ }^{n}$ be vector functions defined for $z$ in $\Omega_{h}$ and for $n$ such that $0 \leqq n k \leqq T_{1}$; introduce the "spacetime" maximum and $L_{2}$ norms

$$
\begin{aligned}
\|\mathbf{u}\|_{\max , T_{1}} & =\max _{0 \leqq n k<T_{1}}\left\|\mathbf{u}^{n}\right\|_{\operatorname{mix}} \\
\|\mathbf{u}\|_{T_{1}} & =\max _{0 \leqq n k<T_{1}}\left\|\mathbf{u}^{n}\right\| .
\end{aligned}
$$

The equations 


$$
\begin{aligned}
\left(I-k Q_{1}\left(\omega^{n}\right)\right) \mathbf{u}^{n+1 / 3} & =\mathbf{u}^{n}, \\
\left(I-k Q_{2}\left(\omega^{n}\right)\right) \mathbf{u}^{n+2 / 3} & =\mathbf{u}^{n+1 / 3}, \\
\mathbf{u}^{n+1} & =P\left(\mathbf{u}^{n+2 / 3}+k \mathbf{E}^{n+1}\right), \\
\mathbf{u}^{0} \text { given }, &
\end{aligned}
$$

define a mapping $\boldsymbol{\omega} \rightarrow \mathbf{u}$. This mapping maps the maximum norm sphere

$$
\|\omega\|_{\max , T_{1}} \leqq 2\|\mathrm{w}\|_{\max , T_{1}}
$$

into the $L_{2}$ norm sphere

$$
\|\mathrm{u}-\mathbf{w}\|_{T_{\mathrm{i}}} \leqq\|\mathrm{w}\|_{\max , T_{1}} h^{l / 2} .
$$

For $\left\|\mathbf{u}^{0}-\mathbf{w}^{0}\right\|, k$ and $h$ sufficiently small, this mapping has a unique fixed point which is the solution of (14) and lies close to $\mathbf{v}$, the solution of (1) and (2).

In our analysis we have neglected the effect of round-off error and of the errors arising from the possibly incomplete iterative evaluation of $\mathbf{u}^{n+1}=$ $P\left(\mathbf{u}^{n+2 / 3}+k \mathbf{E}^{n+1}\right)$. It is obvious, however, that the analysis remains valid if the round-off errors are of order $k^{2}$ and provided $\mathbf{u}^{n+1}$ is approximated by a vector $\left(\mathbf{u}^{n+1}\right)^{*}$ such that $D\left(\mathbf{u}^{n+1}\right)^{*}=O\left(k^{2}\right)$. Furthermore, in the dimensionless variables used in this paper the effect of the Reynolds number $R$ on the error is not in evidence. Clearly $C$ depends on $R$ and increases as $R$ increases; i.e. as $R$ increases $k$ and $h$ have to be reduced for accuracy to be preserved. Finally, it is clear that the results of this section apply to certain other quasi-linear equations besides the NavierStokes equations, provided the boundary conditions are homogeneous. In this sense, our results generalize the work of M. Lees (see e.g. [8]), who considered equations with nonlinear terms of a simpler nature.

The Mixed Initial Value-Boundary Value Problem. The main interest of methods such as those considered in this paper lies in their applicability to mixed initial value-boundary value problems. Schemes similar to (14) have been successfully applied by the author to a variety of such problems (see. e.g. [2]). The convergence proof however, becomes considerably more difficult in the presence of boundaries.

Consider in particular the problem of solving Eqs. (1) and (2) in a domain $\Omega$, with $\mathbf{v}^{0}$ given and with the boundary condition

$$
\mathbf{v}=0 \text { on } \partial \Omega \text {. }
$$

Operators $D$ and $\mathbf{G}$ can be constructed so that the identities (4) and (5) are satisfied and Theorems 1 and 2 hold. $D$ and $\mathbf{G}$ thus constructed employ centered differences except on $\partial \Omega$. On $\partial \Omega$ one-sided first-order differences are used whenever the use of centered differences would require functional values at points outside $\Omega$. The projection $P$ associated with $\mathbf{G}$ and $D$ is orthogonal in the space of functions satisfying (26). The proofs of all these statements take into account the fact that the number of $G$-chains is $2^{l}$ independently of the number of points in the mesh.

Difficulties arise, however, when one approaches the convergence proof proper. It is clear from the proof of Lemma 1 of the last section that, were one to use schemes such as (14), one would have to impose on $\mathbf{u}^{n+1 / 3}, \mathbf{u}^{n+2 / 3}$ inhomogeneous boundary conditions of the form 


$$
\begin{array}{ll}
\mathfrak{u}^{n+1 / 3}=k \mathbf{G} \pi^{n} & \text { on } \partial \Omega_{h} \\
\mathfrak{u}^{n+2 / 3}=k \mathbf{G} \pi^{n} & \text { on } \partial \Omega_{h}
\end{array}
$$

where $\pi^{n}$ is the pressure computed at time $t=n k$. Such a procedure has indeed been followed in practice. Unfortunately, in the presence of inhomogeneous boundary conditions the author has not been able to establish the analogues of Lemmas 3 and 4. Moreover, the construction of $\mathbf{w}$ in Lemma 1 does not carry over to the present problem, since $\mathbf{w}$, as given in the last section, does not satisfy the imposed boundary conditions. Both difficulties stem from the fact that in the presence of boundaries the operators $P$ and $\nabla^{2}$ (linear part of $\mathscr{F}$ ) do not commute. This is reminiscent of other situations in numerical analysis where the noncommutativity of certain operators hinders the analysis of fractional-step methods without detracting from their practical usefulness.

It is nevertheless possible to develop schemes for which convergence in the $L_{2}$ norm can be established. As an example, consider the following scheme with two fractional steps:

$$
\begin{aligned}
& u_{i}^{n+1 / 2}=u_{i}^{n}-k \sum_{\beta}\left\{\frac{1}{2}\left(S_{+\beta}+S_{-\beta}\right)\left(u_{\beta}{ }^{n} D_{0 \beta} u_{i}{ }^{n+1 / 2}\right)\right. \\
& \left.+D_{+\beta} D_{-\beta} u_{i}{ }^{n+1 / 2}\right\} \text { in } \Omega_{h}{ }^{0}
\end{aligned}
$$

It is clear the homogeneous boundary condition (28b) contains an error of order $k$. However, since the number of mesh-points on the boundary is $O(h)$ times the number of mesh-points in the whole domain, some accuracy in the $L_{2}$ norm will be preserved. We shall indicate how one can establish that in the $L_{2}$ norm the solution of (28) converges to the solution of the Navier-Stokes equations which satisfies the correct boundary conditions. $\mathbf{u}^{n}$, as given by (28), therefore assumes the imposed boundary conditions in a weak sense. It is clear that the estimates we shall derive will not do justice to the accuracy of the method.

One can verify the following identity

$$
\left(f, \sum_{\beta} \frac{1}{2}\left(S_{+\beta}+S_{-\beta}\right)\left(u_{\beta}{ }^{n} D_{0 \beta} f\right)\right)=0
$$

which holds for all $f$ provided $D \mathfrak{u}=0$ in $\Omega_{h}$ and $\mathfrak{u}=0$ on the boundary. This of course is a discrete analogue of the identity $\int_{\Omega} f u_{j} \partial_{j} f d x=0$, which holds whenever $\operatorname{div} \mathfrak{u}=0$ in $\Omega$ and $\mathbf{u}=0$ on the boundary. Using this identity we can establish the following inequalities:

$$
\left\|\mathbf{u}^{n+1 / 2}\right\| \leqq\left\|\mathbf{u}^{n}\right\|
$$

and

$$
\begin{aligned}
\left\|\mathbf{u}^{n+1}\right\| & \leqq\left\|\mathbf{u}^{n}\right\|+k\left\|P \mathbf{E}^{n+1}\right\| \\
& \leqq\left\|\mathbf{u}^{0}\right\|+k \sum_{i=0}^{n+1}\left\|P \mathbf{E}^{i}\right\| .
\end{aligned}
$$

If we assume that Eqs. (1) and (2) have a solution $\mathbf{v}$ with continuous derivatives up to order four, this inequality can be used to show that if $k=O\left(h^{2}\right)$ 


$$
\left\|\mathbf{u}^{n}-\mathbf{v}^{n}\right\| \leqq \text { constant } \sqrt{ } h, \quad 0 \leqq n k \leqq T .
$$

For two-dimensional problems one can replace (28a) by an explicit scheme (which does not require intermediate boundary data such as (28b)). For small enough Reynolds number and provided $k<h^{2} / 4$ one can then derive an estimate similar to (29). Furthermore, the scheme (28) can be modified so that a convergence proof of the Krzywicki-Ladyzhenskaya type becomes possible.

Since neither the scheme (28) nor its modifications are of any particular practical significance, details and proofs are omitted. (It should be pointed out however, that the system of linear equations (28a) can be solved by successive relaxation, provided the relaxation factor $\omega$ is sufficiently small. For proof, see [9].)

In ending, the author would like to make some comments on the preceding proofs. First of all, he would like to state his belief that the value of a scheme such as (14) lies in its practical usefulness, not in the possible existence of a convergence proof. The value of the convergence proofs lies in the fact that they contribute to the understanding of the numerical processes performed on the computer.

The proof of this paper requires the existence of four or five continuous derivatives of $\mathbf{v}$ and $p$. Furthermore, the error increases as the bounds on the required derivatives increase. This situation is inherent in the very nature of difference schemes; as a result, it is highly improbable that a flow containing a strong cascade process, i.e. a process in which energy is transferred from large to small eddies, can be adequately described by a difference method, for indeed, such flows are characterized by rapid increase in the higher derivatives. This of course excludes turbulence from the range of application of difference methods.

Finally, it has been claimed by several authors that the nonlinear terms in the Navier-Stokes equations must always be cast in "conservation law" form, i.e. in a form which implies the existence of identities for the momentum similar in appearance to those which hold for the solutions of the differential equations. The author knows of no good reason for following this procedure in problems with a smooth solution and has not endeavored to do so.

Courant Institute of Mathematical Sciences

New York University

251 Mercer Street

New York, New York 10012

1. A. J. CHORIN, "The numerical solution of the Navier-Stokes equations for an incompressible fluid," Bull. Amer. Math. Soc., v. 73, 1967, pp. 928-931. MR 35 \#7643. 1968 .

2. A. J. Chorin, "Numerical solution of the Navier-Stokes equations," Math. Comp., v. 22,

3. A. Krzywicki \& O. A. Ladyzhenskaya, "A grid method for the Navier-Stokes equations," Soviet Phys. Dokl., v. 11, 1966, p. 212.

4. R. TEMaM, "Une méthode d'approximation de la solution des équations de Navier-Stokes," Bull. Soc. Math. France. (To appear.)

5. R. Temam, "Sur l'approximation de la solution des équations de Navier-Stokes par la méthode des pas fractionnaires," Arch. Rational Mech. Anal. (To appear.)

6. H. FujITA \& T. Ka'TO, "On the Navier-Stokes initial value problem," Arch. Rational Mech. Anal., v. 16, 1964, pp. 269-315. MR 29 \#3774.

7. O. A. Ladyzhenskaya, Mathematical Problems in the Dynamics of a Viscous Incompressible Flow, Fizmatgiz, Moscow, 1961; English transl., Gordon \& Breach, New York, 1963. MR 27 $\# 5034 \mathrm{a}, \mathrm{b}$.

8. M. LeEs, "Energy inequalities for the solution of differential equations," Trans. Amer. Math. Soc., v. 94, 1960, pp. 58-73. MR $22 \# 4875$.

9. A. J. ChORIN \& O. Widlund, "On the convergence of relaxation methods." (To appear.) 